\title{
Fabrication of closed-pore inclusive low-permittivity substrates insensitive to ambient humidity
}

\author{
Miyo WAKIYAMA, Kiminori WAKU, Hidetaka HAYASHI and Akira KISHIMOTO \\ Division of Chemistry and Biochemistry, Graduate School of Natural Science and Technology, Okayama University, \\ 3-1-1, Tsushima-naka, Kita-ku, Okayama 700-8530
}

\begin{abstract}
With the aim to reduce the permittivity of a dielectric substrate, closed pores have been introduced into alumina based ceramics by our innovated superplastisically foaming method. Affect of the ambient humidity on the dielectric constant was compared to those for conventional porous ceramics as well as full densified one. In the dry atmosphere, the dielectric constant (k) decreased with the porosity similar to the conventional porous ceramics. Affect of the ambient humidity on the dielectric property was almost identical to that of fully densified one. The degradation of mechanical strength depended on the pore size introduced.
\end{abstract}

(C2009 The Ceramic Society of Japan. All rights reserved.

Key-words : Alumina, Dielectric constant, Closed pore, Superplasticity, Humidity

[Received April 27, 2009; Accepted June 18, 2009]

\section{Introduction}

In the modern information-driven society, high-speed data transmission is required in order to efficiently exchange everincreasing quantities of information. As integrated circuits are reduced in size, gains in speed are limited by increases in resistive-capacitive (RC) delay. An integrated circuit (IC) has multiple closely spaced conductive pathways, carrying electrical signals. As the parasitic capacitance between these IC pathways varies inversely to their spacing, the delay between signal input and signal output (RC delay) arising from parasitic capacitance is becoming ever more problematic with shrinking circuit sizes. To overcome this phenomenon, it is effective to reduce the capacitance between the conductive paths by employing a low dielectric constant (low- $k$ ) material to separate the conductors. Whilst a wide variety of low- $k$ dielectric materials have been proposed for this purpose, ${ }^{1)-8)}$ there are few suitable substrate candidates, many of the proposed materials having insufficient mechanical strength.

An effective strategy for reducing the overall dielectric constant is to introduce hetero materials with low permittivity into the usual chip substrate. ${ }^{7,8)}$ Gas phase materials are promising candidates because their dielectric constants are usually small, i.e., the dielectric constant of air is almost identical to that of vacuum. Thus the introduction of gas-filled pores into a chip substrate may be effective in reducing its electrical permittivity. Reactivity between introduced hetero materials and the substrate matrix may be considered negligible.

When porosity is increased during the usual fabrication method for porous ceramics, the population of connected pores becomes large, resulting in the formation of an open-pore network. In such cases, ambient humidity might adversely affect the dielectric constant as water becomes adsorbed onto the enlarged available surface area $\left(\varepsilon_{\mathrm{r} \text { water }}=80\right) \cdot{ }^{9-12)}$. Mechanical strength is usually degraded substantially by increasing the porosity, leading

Corresponding author: A. Kishimoto; E-mail: kishim-a@cc. okayama-u.ac.jp to diminishing the original substrate role or maintaining the conducting path stably.

We have previously fabricated a novel porous ceramic by expanding pores within fully densified ceramics ${ }^{13)-22)}$ via their superplastic deformation, driven by gas evolution from a previously introduced foaming agent, usually SiC. The conventional fabrication method for porous ceramics uses previously introduced pore-forming materials, which are then decomposed prior to sintering so as to maintain the void space formed during decomposition. The degree of porosity obtained through this method is small with sintering carried out to completion. To obtain a large degree of porosity, only partial sintering should be carried out. Our method, in comparison, can enhance the degree of porosity whilst maintaining dense pore walls as it only selectively introduces closed pores and a high degree of porosity. Furthermore, the fully densified matrix should give rise to favorable mechanical properties. ${ }^{21)}$

With the aim of fabricating a high-frequency dielectric substrate, we investigated methods to fabricate a porous aluminabased substrate containing only closed pores, insensitive to ambient humidity. We also conducted preliminary examination of the material's mechanical strength.

\section{Experimental}

Composite granules composed of foaming agent $(\beta$-SiC, $\mathrm{ABCR}, \mathrm{GmbH} \& \mathrm{Co}$., Germany) surrounded by alumina (AKP30, Sumitomo Chemical, Co.) were prepared. After compaction followed by sintering, the superplastically foamed porous ceramics contained only closed pores. We have previously fabricated a macroscopic alumina monofoam containing only one pore in a pellet (pellet $\varnothing=20 \mathrm{~mm}$ ) by dispersing $3 \mathrm{~mol} \%$ yttria stabilized zirconia (3YSZ) as an agent to facilitate superplasticity. ${ }^{16), 19)}$ Based on this study, alumina powder containing $30 \mathrm{~mol} \% 3 \mathrm{YSZ}$ was chosen to give a matrix with maximized porosity from a constant quantity of foaming agent.

As shown in Fig. 1, two kinds of composite powder granules having different $\beta$-SiC core sizes were prepared. ${ }^{18), 21)}$ Fifteen $\mathrm{wt} \%$ of $\beta$-SiC powder was dispersed in a $1 \mathrm{wt} \%$ methylcellulose 

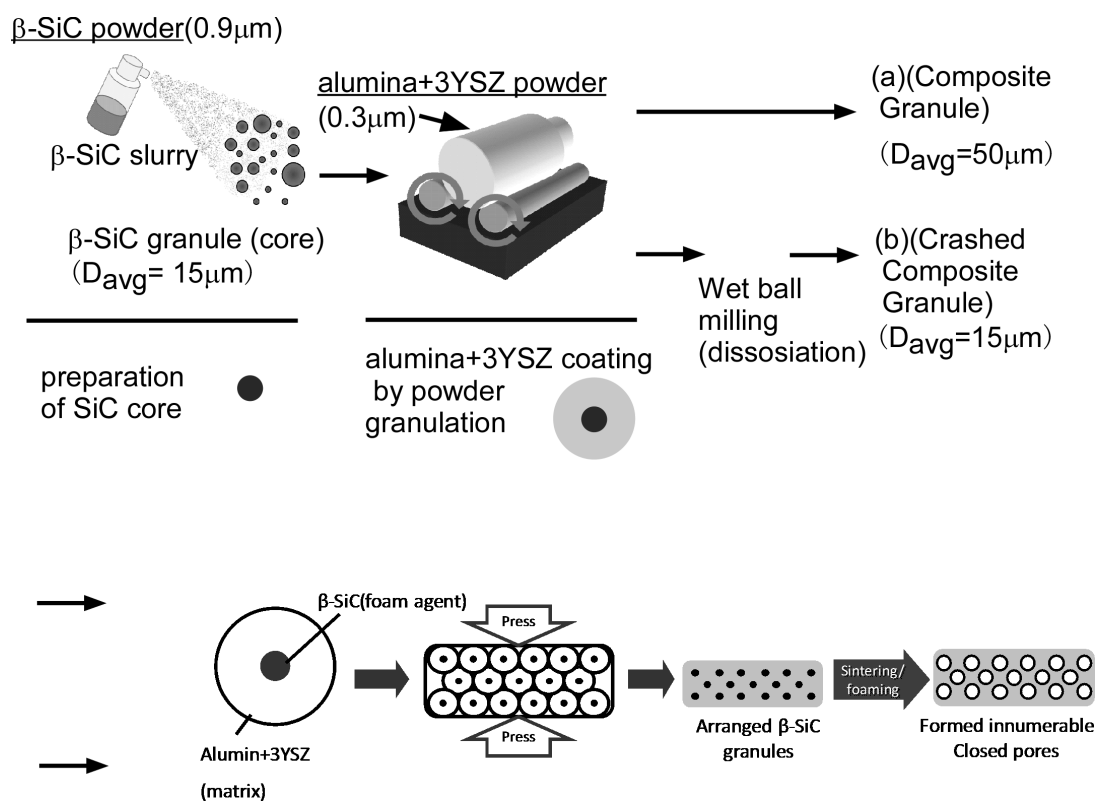

Fig. 1. Schematic illustration to prepare composite granules with different size of $\beta$-SiC cores coated by matrix alumina dispersed with 3 YSZ.

aqueous solution to make a low-viscosity slurry. By spraying this slurry on the matrix powder and then ball milling the resultant mixture, composite granules with a core size of $15 \mu \mathrm{m}$ were fabricated (composite granule (a)).

The composite granules (a) were crushed in a planetary ball mill to give flakes having cores of $<3 \mu \mathrm{m}$ in size partially connected to matrix powder granules (crushed composite granule (b)).

The two kinds of composite granule (a) and (b) were pressed uniaxially in a $\varnothing 20 \mathrm{~mm}$ steel die at $31 \mathrm{MPa}$, and then pressed hydrostatically at $124 \mathrm{MPa}$. The resulting powder compacts were heated at $1600^{\circ} \mathrm{C}$ for $8 \mathrm{~h}$ to sinter them following foaming. The size of the resultant porous pellets differed with their porosity, ranging from 16 to $19 \mathrm{~mm}$ in diameter and 2.5 to $3.5 \mathrm{~mm}$ in thickness.

Fully densified ceramics using the same matrix materials or 30 mol\% 3YSZ dispersed alumina and conventional porous ceramics with porosity controlled by compaction pressure, were also fabricated using the same temperature regime, to act as reference materials.

The apparent density was measured with by Archimedes method using water as the medium. Cross sections were observed by optical or scanning electron microscopy (S-4300, Hitachi, Ltd.). Crystalline phases formed were identified by Xray diffraction (XRD) (Multiflex, Rigaku Co.).

Silver paste was painted on the top and bottom faces of a disc specimen and heat treated at $\sim 300^{\circ} \mathrm{C}$ for $1 \mathrm{~h}$ to form parallel electrodes with a diameter of $10 \mathrm{~mm}$. The AC electric capacitance at $100 \mathrm{~Hz}$ was measured with an impedance analyzer (HP4192A, Palo Alto, USA) and the dielectric constant was determined from the capacitance, sample thickness, and electrode area.

The dependence of the dielectric constant on the ambient humidity was evaluated using two different methods. One method modeled the effects of condensation occurring on a cooled capacitor exposed to a humid atmosphere. Samples were submerged in distilled water for $10 \mathrm{~min}$., then the surface water was removed and the capacitance measured. The other method aimed to determine the time dependence of the capacitance change upon abruptly changing the capacitor's environment from a dry to a humid atmosphere. The sudden change to a humid atmospheric environment was brought about by bubbling air through water and introducing the damp air stream into a glass tube containing the electrode sample. ${ }^{20)}$

The mechanical strength was measured with a three-point flexure machine (EZ-test, Shimadzu Co.) using a rectangular bar $(2.6 \times 2.0 \times 10 \mathrm{~mm})$ with a span length of $9 \mathrm{~mm}^{20), 21)}$

\section{Results and discussion}

We have previously reported that the porosity of superplastisically foamed ceramics depends upon the deformation speed and limit of the matrix as well as the quantity of gas evolved from the foaming agent. ${ }^{15), 17)}$ In this study, porous alumina-based ceramics with porosities ranging from 7 to $40 \%$ were obtained by changing the matrix:core foaming agent ratio using the same temperature program.

Figure 2 shows SEM micrographs for the two types of superplastically foamed ceramics formed from composite granules (a) and crushed composite granules (b). In all cases, almost spherical pores were introduced having dense pore walls. Pore diameters calculated from the cross sections of ceramics formed from (a) and (b) were $170 \mu \mathrm{m}$ and $50 \mu \mathrm{m}$, respectively. Both pore sizes were larger than their corresponding foaming agent cores ( $15 \mu \mathrm{m}$ and $3 \mu \mathrm{m}$, respectively), indicating pore expansion after the densification of the matrix driven by the gas evolution according to the following active oxidation. ${ }^{13)-15)}$

$$
\mathrm{SiC}+\mathrm{O}_{2} \rightarrow \mathrm{SiO}(\mathrm{g})+\mathrm{CO}(\mathrm{g})
$$

XRD confirmed that the matrix formed from alumina containing $30 \mathrm{~mol} \%$ of zirconia was a composite of alumina and zirconia. Studies have indicated that dispersed zirconia particles act to suppress grain growth and thus facilitate superplasticity, leading to pore expansion. ${ }^{16), 17), 19)}$

Figure 3 illustrates the relationship between the porosity $(\rho)$ and the dielectric constant $(k)$ in the superplastically foamed porous ceramics formed from both composite granules and crashed composite granules. The dielectric constant decreased monotonically with porosity, however, no obvious dependence 

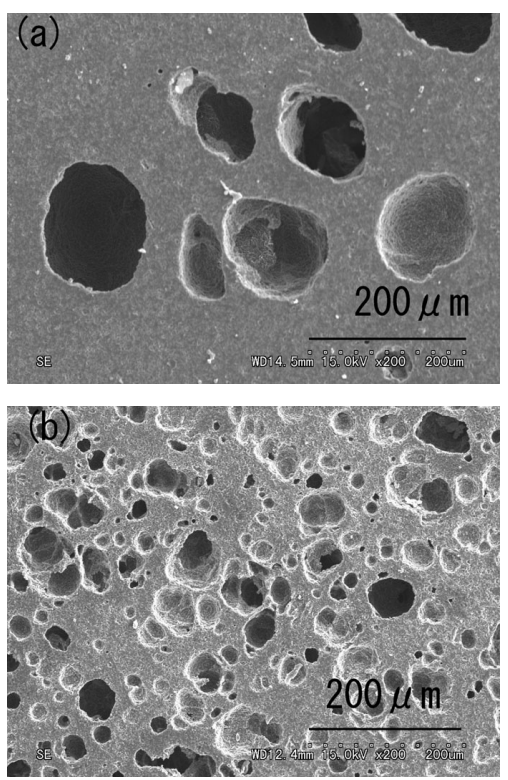

Fig. 2. Scanning electron micrographs for the two types of superplastically foamed ceramics formed from composite granules (a) and crushed composite granules (b).

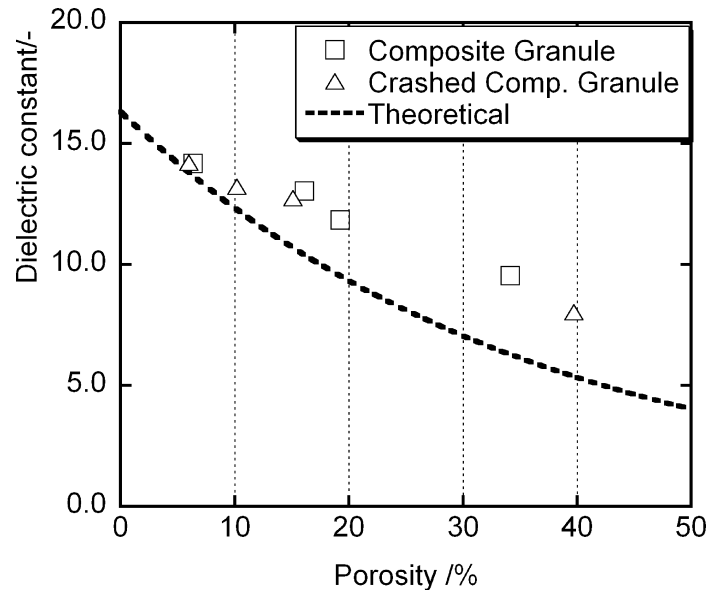

Fig. 3. Porosity dependence of dielectric constant for superplastically foamed alumina based ceramics doped with $30 \mathrm{~mol} \%$ of $3 \mathrm{YSZ}$.

on pore size could be observed. ${ }^{3)-5), 7)}$ Figure 3 also shows two theoretical plots, one based on the logarithmic mixture rule and the other on having the pore/matrix aligned parallel to the longitudinal direction. For the latter case, $k$ decreased linearly with the porosity.

In the case of the logarithmic mixture rule, the dielectric constant decreases suddenly when the pores begin to connect with one another. This occurs because the rule is based on a random distribution of the second phase, the gas-filled pores in this case. In the porous ceramics we created in this study, foaming agents were incorporated such that only closed pores were formed. As such, the matrix remained as a continuous phase above the percolation threshold of around $30 \%$ porosity, leading to a different $k-\rho$ relationship that obeyed the logarithmic mixture rule.

Figure 4 illustrates the change in dielectric constant before and after submersion in water for superplastically foamed, conventional porous, and fully densified ceramics. The degree of porosity for superplastically foamed and conventional porous

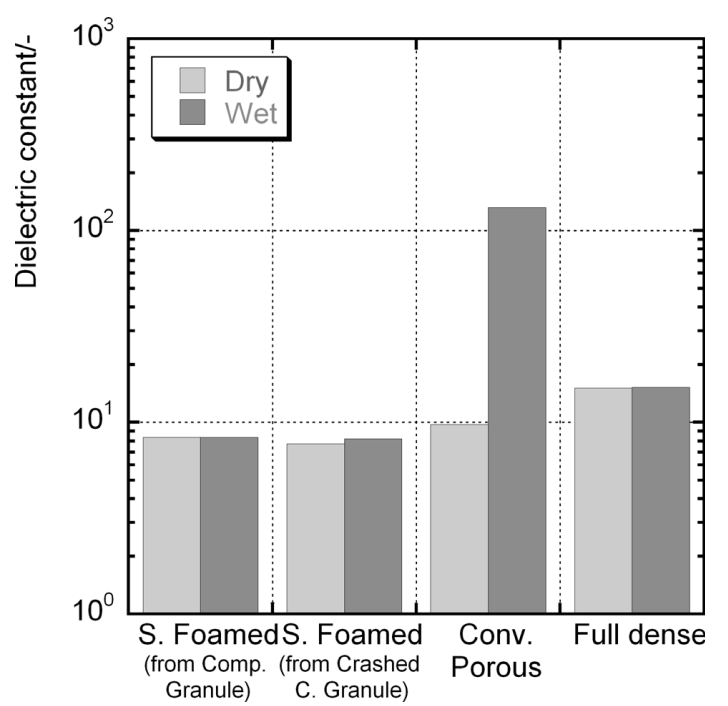

Fig. 4. Dielectric constant of alumina based ceramics with dry and wet conditions.

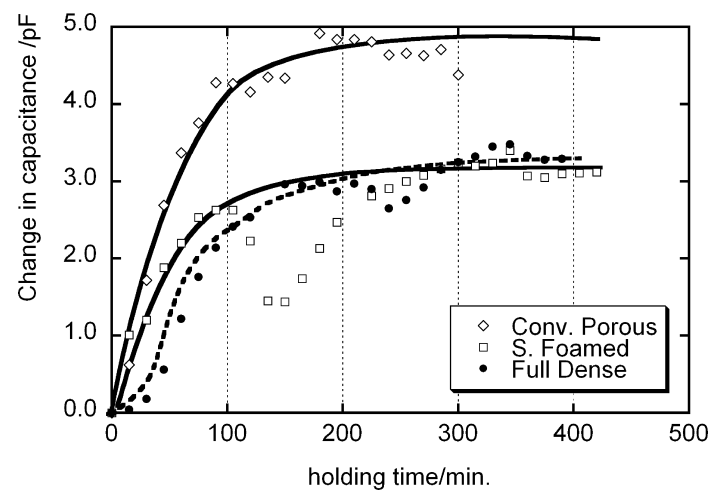

Fig. 5. Change in capacitance for three kinds of alumina based ceramics in humid atmosphere.

ceramics was around $40 \%$. The dielectric constant for the three types of porous ceramics was approximately 8 , or approximately 14 in the case of fully densified ceramics, irrespective of the fabrication method used.

After submersion in water, the dielectric constant of the conventional porous ceramics rose by more than an order of magnitude. By comparison, the increase in dielectric constant for the fully densified porous material was marginal. Since the pores in the conventional porous ceramics, where porosity was over $40 \%$, were open in nature, residual adsorbed water gave rise to the substantial increase in the dielectric constant. Although such porous ceramics achieved a lowered dielectric constant, they were not suitable for practical applications as the dielectric value was likely to increase vastly under condensation forming conditions. $^{4), 9)}$

The superplastically formed porous ceramics exhibited only a negligible change in dielectric constant before and after water submersion, similar to that for the fully densified material. As the introduced pores were all of a closed nature, water infiltration was not possible, resulting in an identical dependence upon humidity for the dielectric constant to that exhibited by the fully densified material. ${ }^{4)}$

Changes in electrical capacitance with time, following the introduction of a high humidity atmosphere, are plotted in Fig. 5. 


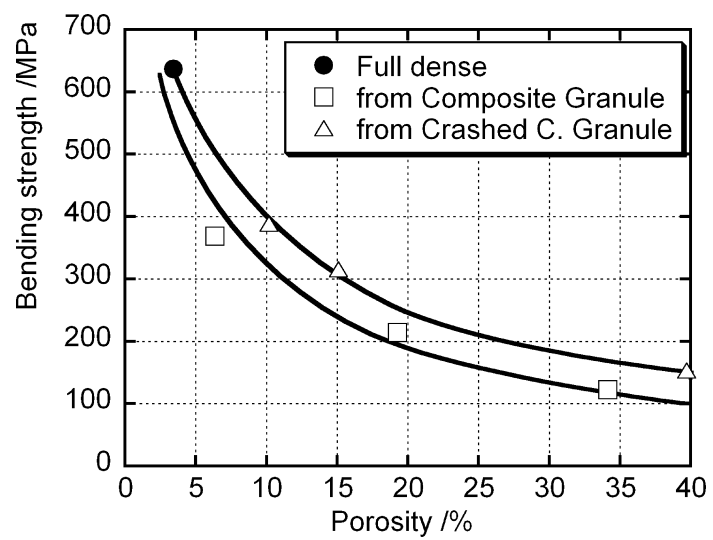

Fig. 6. Porosity dependence of bending strength of superplastically foamed alumina based ceramics with different sized pores.

The ambient humidity surrounding the sample was increased from $40 \%$ relative humidity (RH) to $80 \% \mathrm{RH}$ within ten seconds by introducing water-saturated air at $50 \mathrm{ml} / \mathrm{min}$ into the test chamber (chamber volume $=100 \mathrm{ml}$ ). Although there are some fluctuations, the electrical capacitance tends to increase with the adsorption of water from the introduced high-humidity air. Water adsorption appeared to reach an equilibrium level with the capacitance reaching a constant value. The capacitance gain in the conventional porous ceramic material was 1.5 times that of the fully densified material due to the large available surface area of the conventional material. Korolev et al. $^{22)}$ have also reported increased capacitance in porous alumina upon introduction of saturated water vapor. As the available surface area of the superplastically foamed material was equivalent to that of fully densified substrate, they showed almost identical capacitance gains and profiles for the capacitance $v s$. time plots upon exposure to high RH atmosphere.

Figure 6 shows the relationship between porosity and bending strength for the two types of superplastically foamed ceramics. In both cases, the strength decreased with increasing porosity. The degree of strength degradation for a given degree of porosity was greater for samples formed from composite granules compared with those from crushed composite granules. This may be attributed to the difference in pore sizes, these being three times greater in composite granule-derived materials than in crushed composite granule-derived material. There is scope to further reduce pore size, thus increasing mechanical strength, as even the smaller pores in crushed composite granule derived materials can be as large as $50 \mu \mathrm{m}$ in diameter.

\section{Summary}

With the aim to reduce the permittivity of a dielectric sub- strate, closed pores have been introduced by our innovated superplastisically foaming method. Affect of the ambient humidity was compared to those for conventional porous ceramics as well as fully densified one. The dielectric constant decreased with the porosity similar to the conventional porous ceramics. Affect of the ambient humidity on the dielectric property was almost identical to that of fully densified one. The degradation of mechanical strength depended on the pore size introduced.

\section{References}

1) S. K. JangJean, C. P. Liu, Y. L. Wang, W. S. Hwang, W. T. Tseng, S. W. Chen and K. Y. Lo, Thin Solid Films, 447-448, 674-680 (2004).

2) K. Kim, D. Kwon and G. S. Lee, Thin Solid Films, 332, 369374 (1998).

3) C. Y. Kim, R. Navamathavan, H. J. Lee and C. K. Choi, Surf. Coat. Technol., 202, 5688-5692 (2008).

4) C. J. Chang, M. S. Wu and P. C. Kao, Micropor. Mesopor. Mater., 111, 267-275 (2008).

5) S. Y. Chang, S. M. Jang, S. J. Lin and M. S. Liang, Thin Solid Films, 466, 54-61 (2004).

6) J. Y. Zhang and I. W. Boyd, Opt. Mater., 9, 251-254 (1998).

7) J. Lin and X. Wang, Polymer, 48, 318-329 (2007).

8) S. A. Moghaddam and N. Masoumi, Microelectronics J., 39, 1751-1760 (2008).

9) A. Iqbal, H. Juneja, J. Yao and F. Shadman, Aiche Journal, 52, $15861592(2006)$

10) C. T. Wang, C. L. Wu, I. C. Chen and Y. H. Huang, Sens. Actuators B-Chem., 107, 402-410 (2005).

11) W. M. Sears, Sens. Actuators B-Chem., 130, 661-667 (2008).

12) F. Korolev, V. G. Kytin, L. Nosova and S. N. Kozlov, Phys. Stat. Sol., 202, 1096-1101 (2005)

13) A. Kishimoto, T. Higashiwada, H. Asaoka and H. Hayashi, Adv. Eng. Mater., 8[8], 708-711 (2006).

14) A. Kishimoto, T. Higashiwada, M. Takahara and H. Hayashi, Mater. Sci. Forum, 544-545, 641-644 (2007).

15) T. Higashiwada, H. Asaoka, H. Hayashi and A. Kishimoto, $J$. Eur. Ceram. Soc., 27, 2217-2222 (2007).

16) A. Kishimoto, M. Obata and H. Hayashi, J. Eur. Ceram. Soc., 27, 41-45 (2007).

17) Y. Hashida, H. Hayashi and A. Kishimoto, J. Jpn. Soc. Powder Powder Metallurgy, 54, 740-743 (2007).

18) M. Hanao, H. Hayashi and A. Kishimoto, J. Jpn. Soc. Powder Powder Metallurgy, 54, 732-737 (2008).

19) M. Obata, A. Kishimoto and H. Hayashi, J. Alloys. Compd., 471, 32-35 (2009).

20) A. Kishimoto, M. Obata, K. Waku and H. Hayashi, Ceram. Int., 35, 1441-1445 (2009).

21) A. Kishimoto, M. Hanao and H. Hayashi, Adv. Eng. Mater., 11[1-2], 96-100 (2009).

22) Y. Nishino, H. Hayashi and A. Kishimoto, J. Jpn. Soc. Powder Powder Metallurgy, 56, 389-394 (2009). 